\title{
Preliminary Study of a New Dynamic Technique to Prevent Water Coning
}

\author{
Renard G., Palmgren Cl., Gadelle C., Lesage J., \\ Zaitoun A., Corlay Ph., Chauveteau G.
}

IFP, France

\begin{abstract}
Copyright 1995 , Steering Committee of the European IOR - Symposium.
This paper was presented at the 8th. European IOR - Symposium in Vienna. Austria, May 16 - 17, 1985

This paper was selected for presentation by the Steering Committee, following review of information contained in an abstract submitted by the author(s). The paper, as presented has not been reviewed by the Steering Committee.
\end{abstract}

\begin{abstract}
In many oil (and gas) reservoirs throughout the world, oil recovery is strongly affected by the presence of a bottom aquifer. The drawdown imposed in production wells induces the water to rise forming a cone. Sooner or later water breaks through, and thereafter the production is characterized by an increasing watercut. In the last years, besides the conventional means of correcting coning effects (smaller well spacing, optimising completion intervals, implementing horizontal well technology), various treatment techniques have been proposed to mitigate coning. However, up to now, none of these techniques have proved successful.
\end{abstract}

The purpose of this paper is to present the bases of a new technique to prevent coning. As opposed to current techniques that generally focus on the near-wellbore region after water breakthrough, the new preventive technique is intended to delay breakthrough. It is a dynamic technique consisting of injecting a product, soluble or dispersed in water, into the aquifer. When the product comes into contact with the oil, an impervious barrier to water is formed. The continuing presence of the product in the aquifer provides a self-adjusting process. During injection, oil production could be maintained.

Laboratory tests dealing with product characterization, product injectivity and oil/product interaction are currently performed. Nume- rical simulations have been carried out to study the movement of the product, the generation of a dynamic barrier, and the efficiency of such a dynamic barrier under a wide variety of operating and reservoir conditions. Calculations show that the in-situ spread is an important aspect in optimizing the process. In the case of a homogeneous aquifer zone, simulations show that the effective radius attained by the product is considerable and that oil recovery closely related to this radius is dramatically improved. Not only is the watercut strongly decreased but also the oil recovery is improved.

\section{INTRODUCTION}

A great deal of oil (and gas) reserves in the world are found in reservoirs that are underlain by a bottom aquifer. When a well drilled in the pay zone is put on production, it experiences more or less rapidly what is defined as the coning phenomenon. Water rises from the water leg to the perforated interval and breaks through at the well. Once water has entered the well, water production increases with the detrimental consequences of declining oil production and higher operating costs. Generally, only a few percent of the oil in place can be recovered before excessive amounts of water in the production necessitate to abandon the well. From a theoretical point of view, coning tendencies are inversely proportional to the density difference between oil and water and are 
directly proportional to oil viscosity ${ }^{1}$. Another factor which contributes to coning is a large drawdown near the wellbore.

Various strategies have been suggested to ... optimize ...oil. production . in - reservoirs subjected to coning: 1) rate/pressure, 2) perforation/spacing, 3) horizontal well, 4) special methods or techniques.

Most of special techniques proposed to overcome or at least delay water coning in oil wells are based on modifying reservoir properties or water mobility in order to reduce the watercut while maintaining or even increasing the oil production. Treatments involve the injection of chemicals or gas. Once in place these products will act against the flow of water through porous media by modifying either rock or water properties with preferably no alteration of the flow of oil.

Roughly, anti-water-coning techniques can be divided into two groups by both the volume of fluids to inject and the distance away from the well that these fluids can cover. The first group consists of local well treatments. A limited amount of an agent (polymer, gas, etc.) is injected through the perforated interval. In situ fluid characteristics are mostly changed so that effective water mobility is reduced without or as little as possible altering the oil mobility. When the well is put back on production, the watercut is lower than before the treatment. Unfortunately, local treatments are known to have a limited time effect and efficiency. Besides, companies are reluctant to implement them because incorrectly placed fluids can deteriorate well performance. The other group consists of creating a permeability barrier below the well deep into the reservoir to isolate the aquifer from the oil zone by blocking the vertical flow of water. Suggested fluids to create the barrier are solutions of polymer, foams or gels. However, placement of these fluids far from the wellbore is not easy. That explains why techniques promoting fluid flow barriers are not widely used.

This paper presents the bases of a new dynamic technique to delay coning effects that overcomes the problem of fluid placement. First, the efficiency of a static barrier located at the oil-water contact (OWC) is numerically assessed. Then the new technique that consists in dynamically installing a selfadjusting flow barrier along the OWC is presented. Finally, resuits of a numerical simulation study of the new technique are exposed.

\section{EFFICIENCY OF A STATIC IMPERMEABLE BARRIER}

Some authors have explored the benefit that would be gained from the action of an artificial impermeable barrier placed below a producing well to suppress adverse water coning effects ${ }^{2-4}$. However, a detailed study of the efficiency of such a barrier in terms of oil recovery has not been presented so far. The following numerical calculations were performed to assess this efficiency. Moreover, they have served as reference for the numerical modelling of the new dynamic technique.

All simulations presented in this paper were carried out using the chemical version of ATHOS $^{\circledR}$, a finite difference reservoir simulator ${ }^{5}$. The reservoir is assumed homogeneous and horizontal, the oil zone being underlain by an active aquifer. Basic reservoir and fluid data are summarized in Table 1 , while relative permeabilities are listed in Table 2.

Table 1: Reservoir and fluid data.

\begin{tabular}{|c|c|}
\hline Characteristic & Value \\
\hline Reservoir depth $\quad(\mathrm{m})$ & 1800. \\
\hline Reservoir pressure $\quad(\mathrm{MPa})$ & 18. \\
\hline Reservoir temperature $\left({ }^{\circ} \mathrm{C}\right)$ & 76. \\
\hline Total thickness, $h_{o}+h_{w}$ (m) & 39. \\
\hline Oil zone thickness, $h_{o}$ & $3-12$ \\
\hline Hor. permeability, $K_{h} \quad\left(\mu \mathrm{m}^{2}\right)$ & 1.5 \\
\hline Vert. permeability, $K_{v} \quad\left(\mu \mathrm{m}^{2}\right)$ & 1.5 \\
\hline Porosity, $\Phi$ & 0.3 \\
\hline Initial oil saturation, $S_{o i}$ & 0.85 \\
\hline Residual oil saturation, $S_{\text {or }}$ & 0.20 \\
\hline Water density, $\rho_{w} \quad\left(\mathrm{~g} / \mathrm{cm}^{3}\right)$ & 1.095 \\
\hline Water compress., $c_{w}$ (1/MPa) & $4.2 \cdot 10^{-4}$ \\
\hline Water viscosity, $\mu_{w} \quad$ (mPa.s) & 0.65 \\
\hline FVF - $B_{w}$ & 1.104 \\
\hline Oil density, $\rho_{o}$ & 0.892 \\
\hline Oil compress., $c_{o}$ & $1.010^{-3}$ \\
\hline Oil viscosity, $\mu_{0}$ & 10.0 \\
\hline FVF - $B_{0}$ & 1.16 \\
\hline
\end{tabular}

Table 2: Water-oil relative permeabilities

\begin{tabular}{|c|c|c|}
\hline Water saturation & Krw & Kro \\
\hline 0.15 & 0.000 & 1.00 \\
0.20 & 0.005 & 0.70 \\
0.30 & 0.010 & 0.35 \\
0.40 & 0.030 & 0.20 \\
0.50 & 0.050 & 0.11 \\
0.60 & 0.080 & 0.05 \\
0.70 & 0.140 & 0.02 \\
0.80 & 0.200 & 0.00 \\
\hline
\end{tabular}

Capillary effects are neglected so that there 
is a sharp oil-water contact (OWC) as usually observed in large permeability medium. As the study has been limited to a vertical well, a fully radial reservoir geometry has been considered (Fig. 1), the well being centrally located in a circular drainage area.

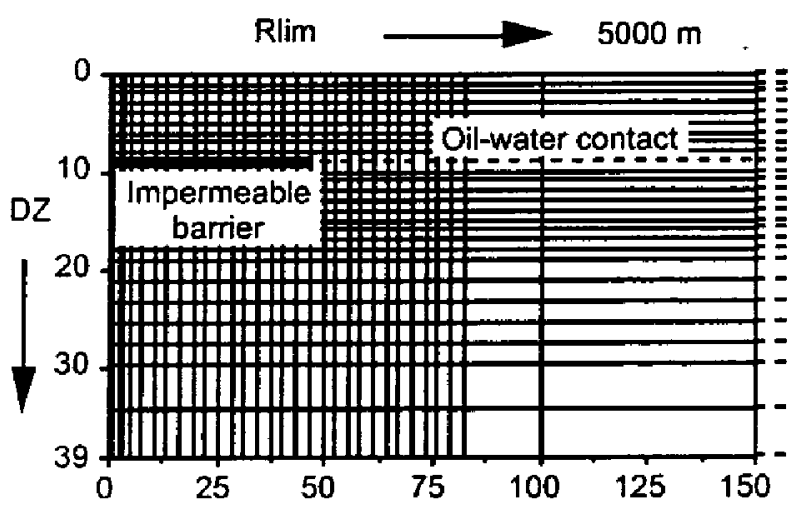

Fig. 1: Radial r $\theta z$ grid used for simulations.

The grid discretization in $r$ and $z$ directions is given in Table 3 . In this section, the artificial barrier assumed static is represented by a flat disk of radius $r_{b}$. The active aquifer is simulated by affecting a large value for the porosity in the cells of the bottom layer of the aquifer.

Table 3: Grid discretization

(radial geometry $-\theta=360^{\circ}$ )

\begin{tabular}{|c|c|}
\hline $\begin{array}{l}\text { Rlim }(\mathrm{m}) \\
\text { From wellbore } \\
\text { center }\end{array}$ & $\begin{array}{l}1.1 .42 .3 .5 .7 . \\
10.13 .16 .19 .22 .25 \\
28.31 .34 .37 .40 .43 . \\
46.49 .52 .55 .58 .61 \\
64.67 .70 .73 .76 .79 . \\
\text { 82. } 100.150 .1000 . \\
5000 .\end{array}$ \\
\hline $\begin{array}{c}D Z(\mathrm{~m}) \\
\text { From top } \\
\text { to bottom }\end{array}$ & $19^{\star 1} \quad 5^{\star} 2 \quad 2 * 5$ \\
\hline
\end{tabular}

The influence of an impermeable (no-flow) barrier was investigated for various oil zone thicknesses, i.e. $3,6,9$ and $12 \mathrm{~m}$, by simulating various lateral extents $r_{b}$ of the barrier, i.e. $0,10,28,46$ and $64 \mathrm{~m}$. As the sum of oil and water thicknesses is constant, an increase in oil thickness corresponds to the same decrease in water thickness. For each oil thickness value, the base case is defined by no artificial barrier. The production well located at the top of the oil zone is open for the first $3 \mathrm{~m}$ ( 3 upper layers). Its total production rate is set to $100 \mathrm{~m}^{3} / \mathrm{d}$ (oil plus water) whatever the oil thickness or barrier radius.

Figure 2 presents typical oil production curves for the case of a $9 \mathrm{~m}$-thick oil reservoir.
These curves indicate that the improvement in recovery due to a static barrier of fixed radius is significant compared to a no flow barrier production.

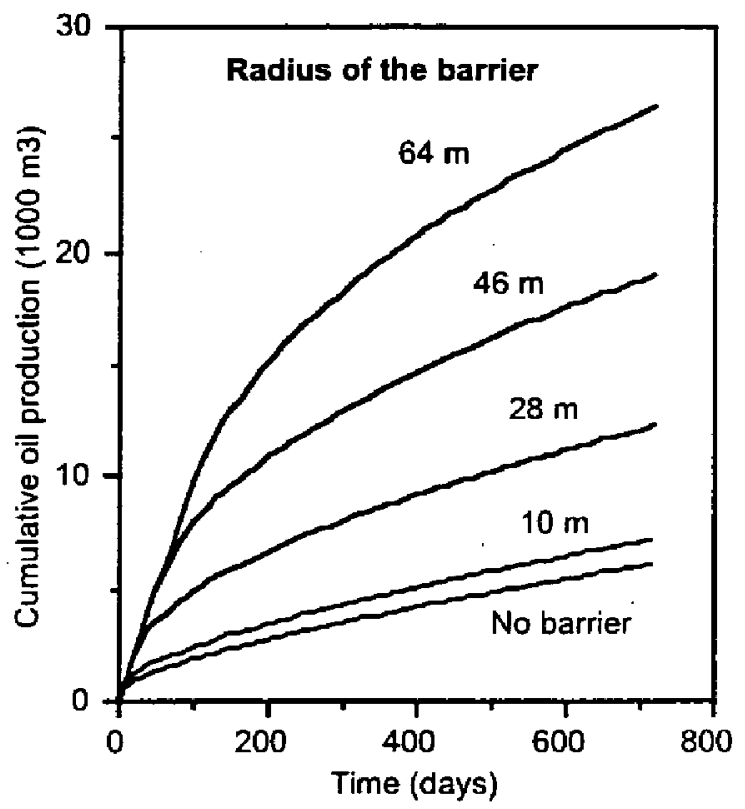

Fig. 2: Typical oil recovery production curves for a 9 m-thick oil reservoir (barrier at OWC).

All runs have been performed on a 2 year period of time. As shown in Figure 3, final watercuts decrease according to the radius of the barrier. Thus, improvement in recovery would continue to increase if simulations were pursued for a longer period of time.

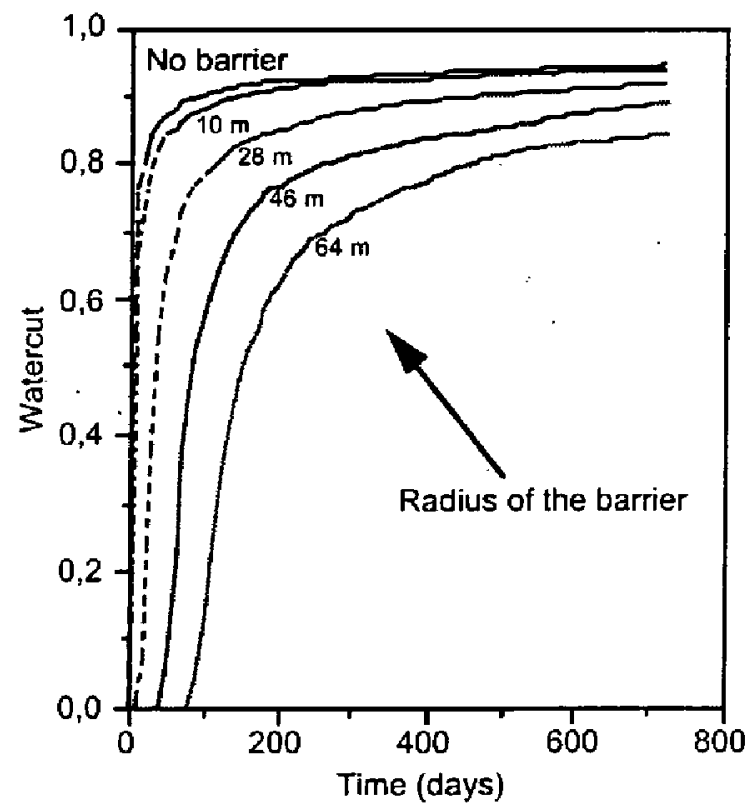

Fig. 3: Watercut versus time for a 9 m-thick oil reservoir (barrier at the OWC).

The increase in oil recovery due to a barrier of given radius $r_{b}$ can be expressed by the oil recovery multiplier at a given time $t$, or $\operatorname{ORM}\left(r_{b}\right)$, defined as the ratio of oil recovery in the presence of the barrier, $N p\left(r_{b}\right)$, to the 
oil recovery without any barrier, $N p\left(r_{b}=0\right)$. According to this definition $O R M(0)=1$. The next table gives the ORM values for different pay zone thicknesses after 2 years of production.

Table 4: $O R M\left(r_{b}\right)$ vs oil zone thickness $h_{0}$ and barrier radius $r_{b}$.

\begin{tabular}{|c|c|c|c|c|c|}
\cline { 3 - 6 } \multicolumn{2}{c|}{} & \multicolumn{4}{|c|}{$r_{b}(\mathrm{~m})$} \\
\hline$h_{\circ}$ & $\begin{array}{c}N p\left(r_{b}=0\right) \\
(\mathrm{m})\end{array}$ & 10 & 28 & 46 & 64 \\
\hline 3 & 1040 & 1.4 & 2.9 & 5.2 & 8.1 \\
\hline 6 & 3046 & 1.3 & 2.5 & 4.1 & 6.0 \\
\hline 9 & 6055 & 1.9 & 2.0 & 3.1 & 4.4 \\
\hline 12 & 9860 & 1.1 & 1.7 & 2.5 & 3.4 \\
\hline
\end{tabular}

These values, plotted in Figure 4, show clearly the tremendous impact of an impermeable barrier located at the oil-water contact with a radius of at least $40 \mathrm{~m}$. For smaller radii, the improvement may be too low to be justified. The efficiency of a static barrier increases as the pay thickness decreases. Nevertheless, the amount of incremental oil is much greater from a thick oil reservoir than from a thin one. For instance, a barrier with a radius of $46 \mathrm{~m}$ gives an extra oil production of $15086 \mathrm{~m}^{3}$ in a $12 \mathrm{~m}$-thick reservoir and only $4378 \mathrm{~m}^{3}$ in a 3 $\mathrm{m}$-thick reservoir while the ORM value is twice as big for the thinner reservoir ( 5.2 in place of 2.5).

Thus, it is clear that water has a better sweep efficiency above the barrier for thin reservoirs than for thicker ones. In thin reservoirs, water travels to the well with a flooding action even in the uppermost part of the oil zone. This is not the case for thicker reservoirs where a great amount of oil above the barrier is bypassed as soon as water invades the wellbore. The barrier provides a better efficiency as it comes closer to the perforations. In fact, it behaves as a screen located in front of a source of light. Its shadow at a certain distance increases as the screen and the light source get closer. This is similar to the result obtained by moving up the barrier into the oil zone. Karp et al ${ }^{2}$ indicated that the production of a well with an impermeable barrier at the bottom of the perforations would be essentially the same as the production of a well which would have a wellbore radius extending out to the barrier radius.

Other results have been obtained varying the oil viscosity as well as the absolute permeability of the porous medium assuming that all other parameters are unchanged.

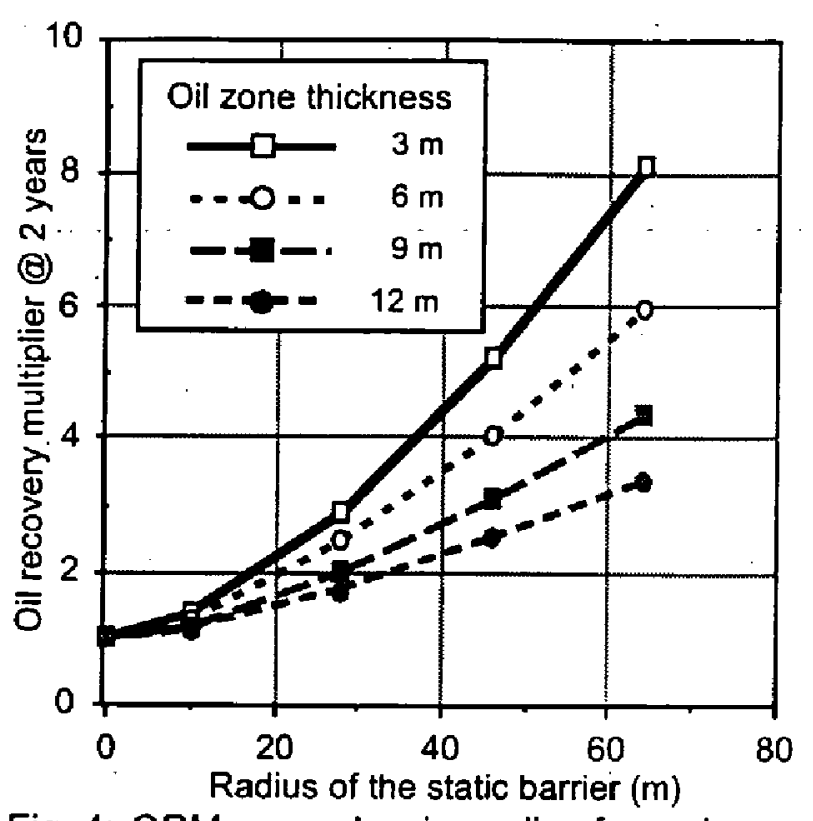

Fig. 4: ORM versus barrier radius for various pay thicknesses (Barrier at the OWC).

The sensitivity to oil viscosity illustrated in Table 5 for a $9 \mathrm{~m}$-thick reservoir and a barrier with a radius of $46 \mathrm{~m}$ shows that the oil recovery multiplier increases with the oil viscosity. On the other hand, the absoiute amount of oil is far greater with a light oil than with a heavy one. On the contrary, the ORM decreases with permeability while the incremental oil increases.

Table 5: Oil production and ORM vs oil viscosity $\left(h_{o}=9 \mathrm{~m}-r_{b}=46 \mathrm{~m}\right)$.

\begin{tabular}{|r|c|c|c|}
\hline $\begin{array}{c}\mu_{o} \\
(\mathrm{cp})\end{array}$ & $\begin{array}{c}N p\left[r_{b}=0\right] \\
\left(\mathrm{m}^{3)}\right.\end{array}$ & $\begin{array}{c}N p\left[r_{b}=46\right] \\
\left(\mathrm{m}^{3)}\right.\end{array}$ & $\begin{array}{c}\text { ORM } \\
{\left[r_{b}=46\right]}\end{array}$ \\
\hline 1 & 27355 & 50002 & 1.8 \\
\hline 10 & 6055 & 18998 & 3.1 \\
\hline 100 & 1543 & 8313 & 5.4 \\
\hline 1000 & 545 & 3566 & 6.5 \\
\hline
\end{tabular}

\section{DESCRIPTION OF A NEW DYNAMIC TECHNIQUE FOR PREVENTING CONING}

Previous simulation results clearly demonstrate that a fluid flow barrier has a tremendous potential for limiting coning effects. However, the main difficulties not solved today are 1) to have a fluid suitable to create such a barrier and 2) to be able to place this fluid deep in the reservoir. Polymer solutions have low injectivity due to their high viscosity. Moreover, they tend to get washed out and prove uneconomical because of the large volumes required to alter reservoir behaviour. In the case of using a gelling system, there will be several challenges to cope with 6 : be sure that the chemicals are stable enough 
and will go where they are intended to (no invasion of the pay zone), deep in the reservoir, and correctly formulated to actually gel, and finally gel after the expected delay. In addition, gels will form an immobile barrier once gelation takes place. If gelation is successful, the gels form an impermeable layer which will necessarily be of limited extent. This severely restricts the efficiency of the barrier due to early water by-passing.

To overcome these problems, a new technique $^{7}$ is proposed to dynamically create the barrier. It is based on the injection, directly in the aquifer, of a chemical solution which becomes unstabilized when contacting oil. The advantages of such materials with respect to polymers or gels are the following:

- The injected solution has a low viscosity and is stable in water for very long times. These properties guarantee a good injectivity for long periods of time, i.e. reduced wellhead pressure during injection. Moreover, they favour a uniform and easy spreading of the solution around the wellbore in the aquifer.

- The destabilization of the solution occurs only when it is in contact with oil. It results in the formation of a locally thin but tight barrier to fluid flow at the oil-water contact.

- The solution that is not yet in contact with oil can continue to flow into the water zone below the barrier penetrating far from the well deeper in the aquifer.

- As soon as water saturated with the active product tries to penetrate the oil zone by finding new flow channels, the active product blocks and stops moving further, dynamically extending the area of the barrier and preventing water influx into the oil zone.

- The formation of the barrier at the OWC prevents the solution to invade the oil zone as opposed to gels or polymers.

The normal procedure to implement the new technique would be to inject the active product in a perforated interval of the well just below the oil-water contact (OWC) while simultaneously producing oil in a second interval at the top of the oil zone. However, this procedure has to be enhanced to spread the active product far enough from the injection source to efficiently block water that sooner or later will be put into movement under the pressure drawdown created by the production well. This is illustrated in the next section that presents a numerical study of this dynamic technique.

\section{NUMERICAL MODELLING OF THE NEW DYNAMIC TECHNIQUE}

\section{1 - Representation of the blocking action}

Simulations of the new technique have been performed with a chemical solution of 2000 ppm concentration. The general behaviour of the blocking product has been mimicked by defining two regions of different mobility reductions. When in the water zone, the product is assumed to be inert with water and rocks (no adsorption) and have the same viscosity and mobility as water. When penetrating the oil zone, the aqueous phase is affected by a mobility reduction factor, $R_{m}$, equal to 200000 as soon as the product concentration is greater than $1500 \mathrm{ppm}$. In the aquifer zone, $R_{m}$ is set to 1 (no mobility reduction).

\section{2 - Problem of fluid placement}

A general problem when injecting a fluid in a partially penetrating well, as is suggested to form the barrier by injecting at the top of the aquifer, is the spreading of the fluid in a hemispherical shape around the injection source. This phenomenon is limited if the aquifer is not too thick or if the injected fluid is much lighter than reservoir brine and much heavier than oil in order to facilitate a natural spreading of the fluid along the OWC. Within the proposed technique, injected fluid would be only slightly lighter than reservoir brine. However, the fluid will react as soon as it is in contact with oil. In this way, only a small volume of the fluid is supposed to penetrate the oil zone. Thus, the volume occupied by the fluid will have a semi-hemispherical shape. The radius invaded by the blocking fluid, proportional to half of the cubic root of the injected volume, will be limited to a few metres away from the well. Table 6 shows the time, in days, required to reach such a radius for various injection rates, $Q_{a p}$, assuming a porosity of 0.3 (Table 1).

Table 6: Time (in days) to reach radius $r$ as a function of injection rate

(Semi-hemispherical flow).

\begin{tabular}{|c|r|r|r|r|}
\cline { 2 - 5 } \multicolumn{1}{c|}{} & \multicolumn{4}{c|}{$r(\mathrm{~m})$} \\
\hline$Q_{3 \rho}\left(\mathrm{m}^{3 / \mathrm{d}}\right)$ & 10 & 20 & 30 & 40 \\
\hline 10 & 63 & 502 & 1696 & 4020 \\
\hline 20 & 31 & 251 & 848 & 2010 \\
\hline 30 & 21 & 167 & 508 & 1340 \\
\hline 50 & 12 & 100 & 339 & 804 \\
\hline 100 & 6 & 50 & 169 & 402 \\
\hline
\end{tabular}

This general behaviour has been confirmed numerically considering a $9 \mathrm{~m}$-thick reservoir 
case. A simulation was run assuming the injection of the blocking product at the top 3 $m$ of the aquifer ( 3 upper layers) at a $50 \mathrm{~m}^{3} / \mathrm{d}$ constant rate. At the same time, a total of 100 $\mathrm{m}^{3} / \mathrm{d}$ oil plus water was produced from the 3 upper layers in the oil zone. Figure 5 shows a map of product concentration after 2 years of simultaneous injection and production (The dark tone corresponds to a concentration level higher than 1800 ppm while light grey indicates absence of the active product).

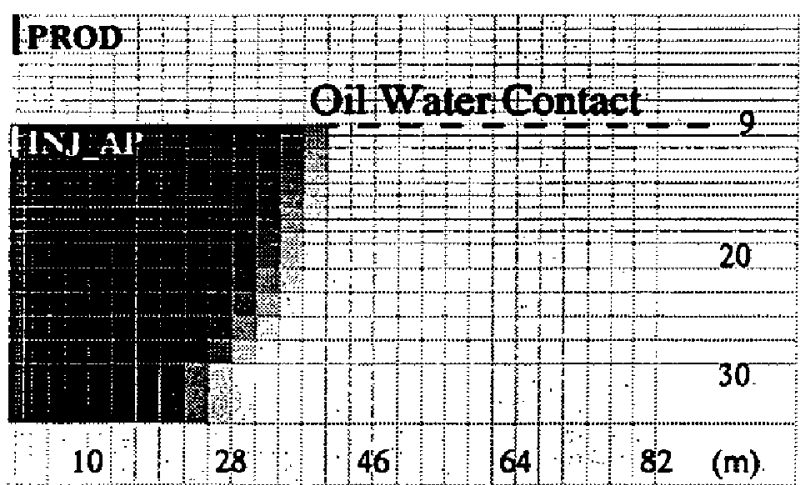

Fig. 5: Injected fluid concentration @ 2 years of simultaneous injection and production.

It is clear that the active product essentially invades the aquifer as previously described. Therefore, its blocking efficiency is low as the barrier has a limited lateral extent, equivalent after two years to that of a fixed barrier with a radius of $28 \mathrm{~m}$ (Fig. 6 - Scenario No 1).

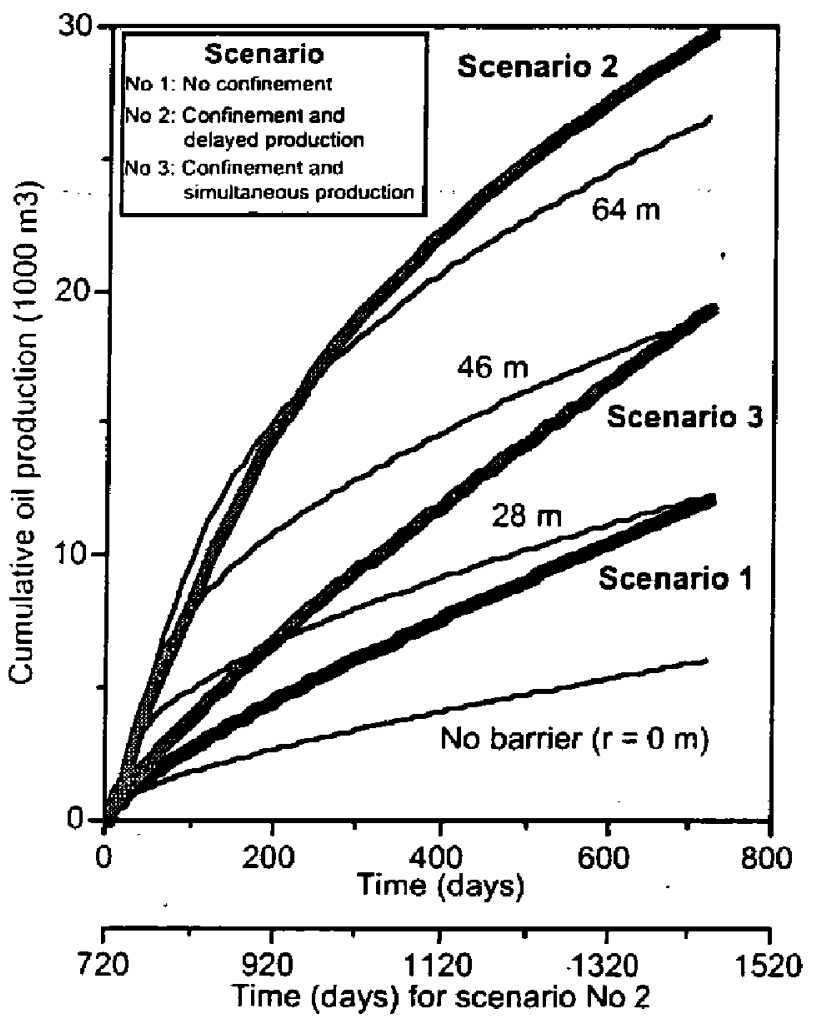

Fig. 6: Efficiency of barriers dynamically placed along the OWC (Curves from Fig. 2 are plotted for comparison).
It has to be noticed that the simultaneous production does not facilitate the fluid spreading (no flux attraction towards lower pressures created by the production). In fact, the region located below the barrier becomes a dead zone in which fluids move too. slowly to enable a good spreading of the solution towards the front where water, free of injected fluid, by-passes the barrier.

\section{3 -Optimization of fluid placement}

It is obvious from the previous calculations that it would take an excessive lapse of time to create an efficient barrier injecting only from one source. Moreover, it would be necessary to inject a very large volume of fluid. To improve the horizontal spreading of the fluid and avoid its downward movement, it is suggested to confine the injection of active product with a simultaneous injection of water a few metres below the fluid injection interval. With such a confinement, it is expected that large distances will be reached by the active product along the oil-water contact while limiting the volume of fluid required to generate the barrier. The following table gives the time required to fill a cylindrical volume with the fluid assuming that the confinement by a water injection limits the thickness of the cylinder to $2 \mathrm{~m}$.

Table 7: Time (days) to reach radius $r$ for various injection rates (Radial flow).

\begin{tabular}{|c|c|c|c|c|}
\cline { 2 - 5 } \multicolumn{1}{c|}{} & \multicolumn{4}{c|}{$r(\mathrm{~m})$} \\
\hline$Q_{a \rho}\left(\mathrm{m}^{3} / \mathrm{d}\right)$ & 10 & 20 & 30 & 40 \\
\hline \hline 10 & 19 & 75 & 167 & 300 \\
\hline 20 & 9.4 & 38 & 85 & 151 \\
\hline 30 & 6.3 & 25 & 56 & 100 \\
\hline 50 & 3.8 & 15 & 34 & 60 \\
\hline 100 & 1.9 & 7.5 & 17 & 30 \\
\hline
\end{tabular}

Several runs were carried out to numerically check the ability of a simultaneous water injection to confine the active product to the very top of the aquifer. Another objective was assigned to these runs: for a given injection rate of the blocking fluid, determination of the rate of water injection that optimizes the placement of this fluid at the OWC. As a simultaneous production will have no influence on the spreading of the injected solution, as previously observed, fluid placement optimization was studied by considering the aquifer zone only. The vertical discretization of the grid for these runs was modified. From the top of the aquifer, thicknesses of layers were: $10^{\star} 1,5^{\star} 2$ and $5^{\star} 5$ metres. The active product is always injected into the 3 upper 
layers of the aquifer while the confining water is injected into a lower non-contiguous interval:: Table 8 summarizes the various parameters used to get the different maps of product concentration shown in Figure 7 after 2 years of dual injection (the dark tone corresponds to a concentration of injected fluid greater than $1800 \mathrm{ppm}$ ). The parameters are the injection rate of confining water, $Q_{c w}$. and the location of the water injection source defined by the indices of its lower $(I)$ and upper layers $(u)$, and the height of the injecting interval $h /$ in metres. The injection rate $Q_{a p}$ of confined fluid is set to $50 \mathrm{~m}^{3} / \mathrm{d}$.

Table 8 : Parameters considered for the various cases shown on Figure 7.

\begin{tabular}{|c|c|c|c|c|}
\hline & \multirow{2}{*}{$\begin{array}{c}\text { Case } \\
\mathrm{Nb}\end{array}$} & $Q_{c w}$ & \multicolumn{3}{|c|}{ Location of } \\
\cline { 3 - 5 } & $\left(\mathrm{m}^{3} / \mathrm{d}\right)$ & $u$ & $l$ & $h /(\mathrm{m})$ \\
\hline 1 & 50 & 12 & 13 & 4 \\
\hline 2 & 100 & 12 & 13 & 4 \\
\hline 3 & 200 & 10 & 13 & 7 \\
\hline 4 & 400 & 7 & 13 & 10 \\
\hline 5 & 600 & 7 & 15 & 14 \\
\hline
\end{tabular}

Figure 7 outlines the increasing lateral spreading of the active product along the oil water contact as the rate of confining water is increased. At the same time, it shows that the zone of dispersion at the front edge of the active product is smaller as the confinement is more severe.

\section{4 - Simulation of oil recovery after fluid placement}

As fluid confinement is effective using another injection source, a full injection production procedure has been simulated on the complete domain (Fig. 1). Two distinct time periods were considered. During the first two years, the active fluid and confining water are injected according to case 4 of Table 8. In the second 2 year period, injectors are shut-in while production takes place at a rate of $100 \mathrm{~m}^{3} / \mathrm{d}$ oil plus water from the 3 upper layers in the oil zone. Oil produced during this second period of time is plotted in Figure 6 , referred to as Scenario No 2 (time zero is defined as the production starts).

The level of production shows that the fluid confinement works pretty well as it allows the dynamic placement of the injected fluid. After 2 years of production, the result of the barrier is equivalent to that of a fixed impermeable barrier extending more than $64 \mathrm{~m}$ along the OWC.

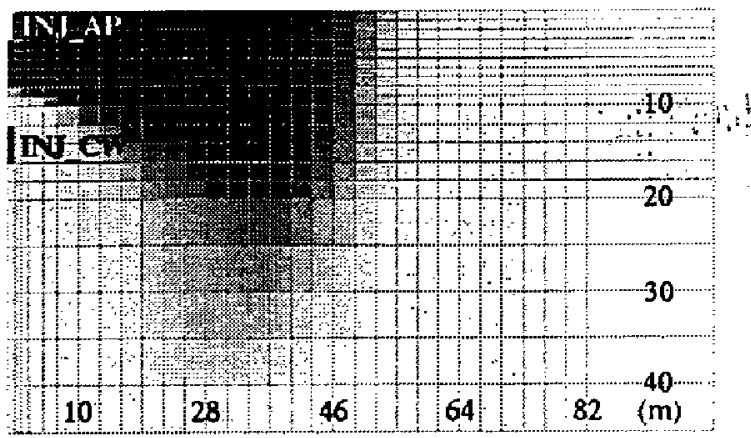

Fig. $7-1: Q_{c w}=50 \mathrm{~m}^{3} / \mathrm{d}$.

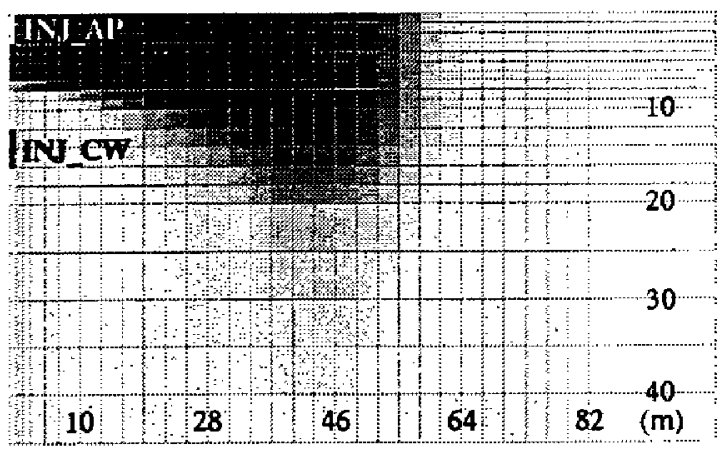

Fig. 7 - 2: $Q_{c w}=100 \mathrm{~m}^{3 /} / \mathrm{d}$.

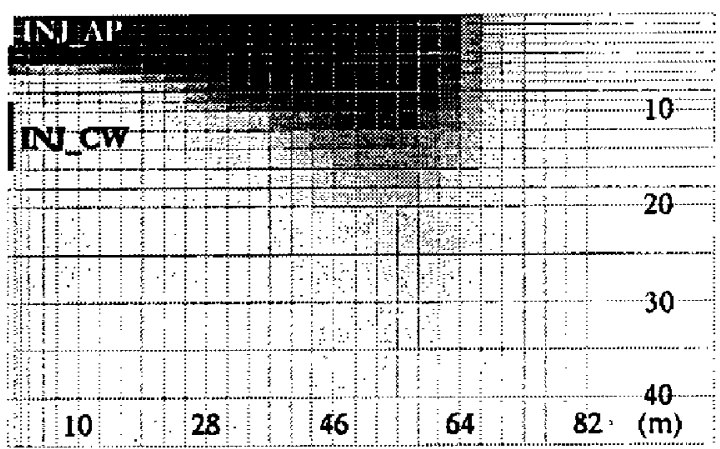

Fig. 7 - 3: $Q_{c w}=200 \mathrm{~m}^{3} / \mathrm{d}$.

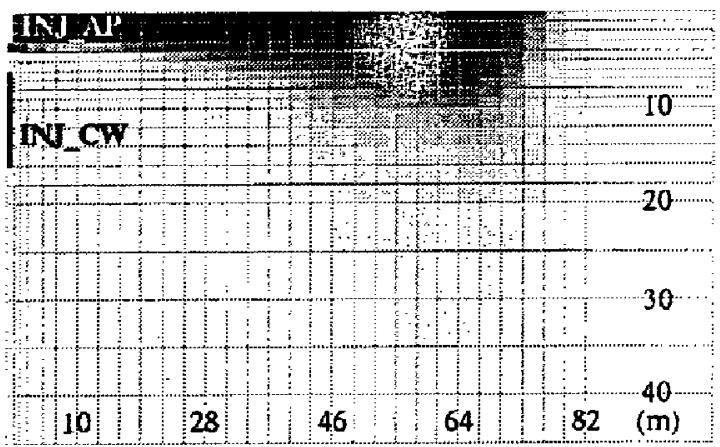

Fig. 7 - 4: $Q_{c w}=400 \mathrm{~m}^{3} / \mathrm{d}$.

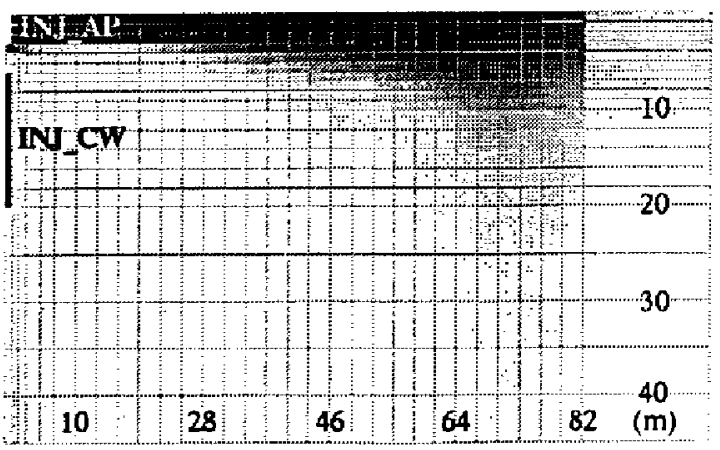

Fig. 7 - 5: $Q_{c w}=600 \mathrm{~m}^{3} / \mathrm{d}$. 


\section{5 - Simulation of simultaneous fluid confi- nement and oil production}

Though it gives the expected result, i.e. a substantial improvement in recovery, the previous procedure presents a severe drawback. Oil production is greatly delayed since production starts only after the placement of the barrier. From a technological point of view, it is a challenge to simultaneously perform the two injections and the production from the same well. However, the triple completion injection/production procedure was evaluated numerically to assess its theoretical potential. The result in terms of oil production is shown in Figure 6 (referred to as Scenario No 3). Recovery is lower than assuming the placement of the barrier prior to production. However, it is similar to that of a fixed barrier with a radius of $46 \mathrm{~m}$, about thrice the recovery without any barrier. Moreover, as for scenario No 2, oil recovery could be pursued for more than 2 years (Fig. 6) since the slope of the production curve has not flattened out at that time. This behavior is due to the selfadjusting barrier that continues to spread along the OWC.

Results obtained considering simultaneous injection and production (Scenarii No $1 \& 3$ ) indicate a poor initial production performance due to the limited effect of the barrier as long as its extent is not big enough. To improve this performance, it would be necessary to start production with some delay compared to the dual injection of the active product and confining water. A possibility, not investigated here, to avoid a triple completion, i.e. dual injection and production, would be to use only one tubing to inject the active product and confining water in alternated slugs. In this case, the tubing would have to be moved at each injection stage in order to be placed in front of the appropriate injection interval, at the top of the aquifer zone to inject the active product and lower in the aquifer to inject the confining water.

\section{PRELIMINARY LABORATORY TESTS}

Some preliminary tests performed in the lab with commercial colloids having a particle size as low as $0.2 \mu \mathrm{m}$ have proven very promising. Propagation of these suspensions in $3 \mu \mathrm{m}^{2}$ sandpacks is excellent with the same pressure drop as with water. Kinetics of destabilization are of several days. Further lab tests with new series of colloidal particle suspensions are carried out to accelerate destabilization kinetics in the presence of oil. Up to now, no tests have been performed combining placement technique and colloid destabilization.

\section{CONCLUSIONS}

Numerical calculations have been performed to assess the impact, in reservoirs underlain by a bottom aquifer, of a static flow barrier located along the oil-water contact. Results indicate that a barrier dramatically improves oil recovery. To quantify the barrier efficiency, the amount of incremental oil due to the barrier is a better criterion than the oil recovery factor.

The bases of a new dynamic technique to delay water coning effects have been presented. The procedure consists to dynamically create a flow barrier at the oil-water contact by injecting a fluid that blocks when it comes into contact with oil but is inert to water and porous medium. This characteristic of the fluid allows to solve the problem of fluid placement that remains the main difficulty with commonly suggested fluids.

Numerical simulations have confirmed the potential of the new technique using dual injection to confine the spreading of the product along the oil-water contact. Production could be accelerated if performed at the same time as the dual injection.

Currently performed laboratory studies should soon give indications on the kinetics of the blocking action of a solution of colloidal particles in contact with oil. From these results, the feasibility of the new technique will be more precisely evaluated. The kinetics of barrier formation are undoubtedly very critical as they are related to the barrier blocking efficiency.

\section{NOMENCLATURE}

$\begin{array}{llr}B_{o} & \text { Oil FVF } & \text { [vol./vol.] } \\ B_{w} & \text { Water FVF } & \text { [vol./vol.] } \\ c_{o} & \text { Oil compressibility } & {[1 / \mathrm{MPa}]} \\ c_{w} & \text { Water compressibility } & \text { [1/MPa] } \\ D Z & \text { Discretization in vertical } & {[\mathrm{m}]} \\ & \text { direction } & {[\mathrm{m}]} \\ h_{o} & \text { Oil zone thickness } & {[\mathrm{m}]} \\ h_{w} & \text { Aquifer thickness } & \\ I n j \_a p & \text { Active product inj. well } & \\ I n j \_c w & \text { Confining water inj. well } & \\ K_{h} & \text { Horizontal permeability } & {\left[\mu \mathrm{m}^{2}\right]} \\ K_{v} & \text { Vertical permeability } & {\left[\mu \mathrm{m}^{2}\right]} \\ N p & \text { Oil production (surface) } & {\left[\mathrm{m}^{3}\right]} \\ O R M & \text { Oil recovery multiplier } & {[-]} \\ P & \text { Reservoir pressure } & {\left[\mathrm{kPa}^{2}\right.}\end{array}$




$\begin{array}{llr}Q_{a p} & \text { Active product inj. rate } & {\left[\mathrm{m}^{3} / \mathrm{d}\right]} \\ Q_{c w} & \text { Confining water inj. rate } & {\left[\mathrm{m}^{3} / \mathrm{d}\right]} \\ \dot{H}_{b} & \text { Barrier radius } & {[\mathrm{m}]} \\ R l i m & \text { Discretization in radial } & {[\mathrm{m}]} \\ & \begin{array}{l}\text { direction } \\ S_{o i}\end{array} & \text { Initial oil saturation } \\ S_{o r} & \text { Residual oil saturation } & {[-]} \\ T & \text { Reservoir temperature } & {[-]} \\ \rho_{o} & \text { Oil density } & {\left[{ }^{\circ} \mathrm{C}\right]} \\ \rho_{w} & \text { Water density } & {\left[\mathrm{g} / \mathrm{cm}^{3}\right]} \\ \Phi & \text { Porosity } & {\left[\mathrm{g} / \mathrm{cm}^{3}\right]} \\ \mu_{o} & \text { Oil viscosity } & {[-]} \\ \mu_{w} & \text { Water viscosity } & {[\mathrm{mPa} . \mathrm{s}]} \\ & & {[\mathrm{mPa} . \mathrm{s}]}\end{array}$

\section{REFERENCES}

1. Muskat, M.: The Flow of Homogeneous Fluids Through Porous Media, McGrawHill Book Co. Inc., New York City (1937); reprinted edition, Intl Human Resources Development Corp., Boston (1982).

2. Karp, J. C., Lowe, D. K. and Marusov, N.: "Horizontal Barriers For Controlling Water Coning," JPT, July 1962, p. 783-790, Trans. AIME, 225.

3. Strickland, R. F.: "An Analysis of Artificial Barriers for Controlling Water Coning," MS Thesis, Texas A\&M University, College Station (1974).

4. Ekrann, S.: "On the Protection Against Coning Provided by Horizontal Barriers of Limited Lateral Extent," 6th European IOR Symposium, Stavanger, May 21-23, 1991, p. 169-178.

5. Lefevre, D. and Labonne, M.: "A Practical Approach to a Software Activity Management - Application to a Multipurpose Reservoir Simulator," SPE 24996, 1992 SPE European Petroleum Conference, Cannes, Nov. 16-18, p. 359-366.

6. Borling, D., Chan, K., Hughes, T. and Sydansk, R.: "Pushing Out the Oil with Conformance Control," Oilfield Review (April 1994) 44-51.

7. Lesage; J., Renard, G., Gadelle, $\mathrm{Cl}$. and Corlay, Ph.: "Procédé et fluide pour l'amélioration de la récupération d'huile dans les réservoirs comportant un aquifère," FR Patent No 2,701,733 (1994). 